\title{
Erratum: Many-body Effects on Dielectric Responses of a Spin-polarized Electron Gas
}

[J. Korean Phys. Soc. 58, 1429 (2011)]

\author{
J. S. KIM, S. PARK and K. S. YI*
}

Department of Physics, Pusan National University, Busan 609-735, Korea

DOI: $10.3938 / \mathrm{jkps} .61 .662$

The acknowledgment should be corrected to 'This work was supported in part for two years by Pusan National University Research Grant (KSY)'. 